\title{
An Improved Algorithm for Indoor Location
}

\author{
Xiao-Feng Liu, Dong-Xu Zhang, Si-Qi An, Xu Sun \\ School of Transportation Science and Engineering Beihang University Beijing, China \\ E-mail: liuxf@buaa.edu.cn, zhangdongxu@buaa.edu.cn, ansiqi@buaa.edu.cn, 854505315@qq.com
}

\begin{abstract}
In order to improve the accuracy and efficiency of indoor location, an improved algorithm of indoor location is proposed in this paper, which is much easier and faster to achieve by a common hardware. The proposed algorithm based on the isosceles triangle layout is much more convenient to extend the area of indoor location than equilateral triangle layout, both of which have been simulated and compared. Considering there is no obvious difference between two algorithms in the ideal computer simulation, hardware simulation is established. Through the hardware simulation, the isosceles triangle layout is proved to be more effective than the equilateral one.
\end{abstract}

Keywords-indoor location; equilateral triangle layout; isosceles triangle layout

\section{INTRODUCTION}

Recently, wireless location has gradually become a hot spot on application in the fields of commercial, military and public security0. It can be used for location, tracking, navigation, logistics guidance and equipment monitoring, automatic motion control, disaster evacuation guidance, battlefield monitoring and environmental observation, etc.

In open areas, the relationship between radio signal intensity and signal propagation distance is exponentially decayed[2]; It is more complex in the indoor environment, there are many walls and other obstacles, coupled with the influence of human activities, resulting in signal transmission of non-line of sight, multipath and other problems, so it is difficult to establish an accurate model. Based on the existing location technology, the precise and stable location and navigation system with high robustness and adaptability can be realized by the means of network communication, wireless information integration and etc. [3]

In 2008, $\mathrm{Xu}$ improved the location accuracy by introducing the difference based on the three-point location, which was verified by hardware experiments 0 . Then, threshold condition, weighted centroid location and the weighted least squares method are proposed to improve the location accuracy [6]. In 2011, Martin developed another WIFI based network application, whose accuracy can be within $1.5 \mathrm{~m}$ [7]. As with WiFiSLAM Company, Martin also used WIFI blot identification technology only with a mobile phone. By 2015, Liu combined equilateral triangle, Gaussian model pretreatment and improved weighted trilateration, significantly improves the location accuracy [8].

The outline of the paper is as follows. Basic concepts and theoretical results of indoor location are presented in Section II. Algorithms and verification algorithm are given in Section III. Algorithms will be verified on a hardware platform in the Section V. Finally, some concluding remarks are stated in Conclusion.

\section{INDOOR WIRELESS LOCATION}

If the distances, $L_{i}, i=1,2,3$, are among an unknown point, $\left(x_{0}, y_{0}\right)$, and three another points, $\left(x_{i}, y_{i}\right), i=1,2,3$, whose coordinates are known. Then the coordinate of unknown point can be inferred. Specific derivation is as follows:

$$
\left(x_{0}-x_{i}\right)^{2}+\left(y_{0}-y_{i}\right)^{2}=L_{i}, i=1,2,3
$$

If $L_{i}$ are accurate, three same points will be coordinated by simultaneous equations. Actually, the distances often contain errors caused by various factors, which leads to the unknown point can't be pinpointed. Trilateration, the angle threshold condition, weighted centroid location, differential location, weighted least squares method and other methods will be adopted to improve the location accuracy.

The proposed indoor wireless location method is based on Received Signal Strength Indicator, RSSI, which calculates distances by received signal strength[9]. Currently, the most popular location method is based on RSSI [9].

Under the open environment, radio wave power loss formulation is given by:

$$
\text { LOSS }=20 \lg \left(\frac{4 \pi d}{\lambda}\right)
$$

where $d$ is the distance between receiving point and source point, and $\lambda$ is radio wave length.

Radio waves logarithmic - normal distribution model is:

$$
\operatorname{LOSS}_{d}=A+10 n * \lg \left(\frac{d}{d_{0}}\right)
$$

where $\operatorname{LOSS}_{d}$ is loss of signal strength, $d$ is the distance between the receiving point and the source point, $d_{0}$ is the reference distance(usually to be 1 meter), $A$ is loss of signal strength at $1 \mathrm{~m}, n$ is loss exponent.

The relationship between the RSSI values and distance is given by:

$$
R S S I=A-10 n * \log (d)
$$


As described above, in practical application, the uncertain environmental factors make it impossible to pinpoint. High-quality equipment or optimized algorithms can be used in order to pinpoint. In this paper, a high reliability hardware will be applied, the main research is to identify better algorithms.

Since the distance calculated by the signal strength is generally larger than the actual value, more than one point will be got. This will bring in the choice of points, which will increase the amount of calculation. The proposed isosceles triangle layout location algorithm can more easily carry out the two points trade-offs, and solve simultaneous equations easier.

\section{ISOSCELES TRIANGLE LAYOUT LOCATION ALGORITHM}

\section{A. Experimental Area}

The experimental area can be extended by docking two identical isosceles right triangle's hypotenuse to a square area. In order to facilitate the actual operation, a square area (shown in Fig.1) is chosen as an experimental area.

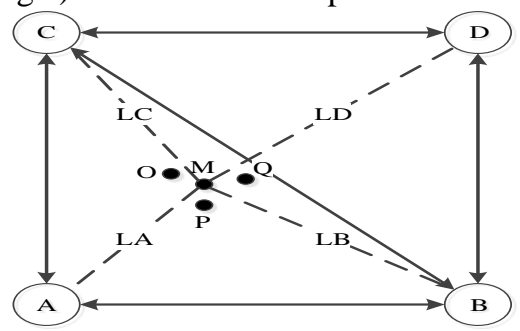

Figure 1. Isosceles right triangle layout location area map.

As the Fig. 1 shows, in the known four points, point A, B and $\mathrm{C}$ form the experimental area $\triangle \mathrm{ABC}$, point $\mathrm{D}, \mathrm{B}$ and $\mathrm{C}$ form the experimental area $\triangle \mathrm{DBC}$, point $\mathrm{M}$ is the unknown point. Point $\mathrm{O}$ is result of solving two simultaneous equations of point $\mathrm{A}$ and $\mathrm{C}$. Point $\mathrm{P}$ is result of solving two simultaneous equations of point $\mathrm{A}$ and $\mathrm{B}$. Point $\mathrm{Q}$ is result of solving two simultaneous equations of point $\mathrm{B}$ and $\mathrm{C}$. Switching condition of two experimental areas is the size relationship of LA (the distance between point A and point $\mathrm{M})$ and $\mathrm{LD}$ (the distance between point $\mathrm{D}$ and point $\mathrm{M}$ ).

Isosceles triangle layout location algorithm involves screening intersection point (SIP). The process of screening intersection point is as follow: In the experimental area of $\triangle \mathrm{ABC}$, in solving two simultaneous equations of point $\mathrm{A}$ and $\mathrm{C}$, a positive abscissa will be taken, and ordinate of two intersection points are identical; In solving two simultaneous equations of point $\mathrm{A}$ and $\mathrm{B}$, a positive ordinate will be taken, and abscissa of two intersection points are identical. In solving two simultaneous equations of point $\mathrm{A}$ and $\mathrm{C}$, the smaller one of two abscissas will be selected; so do ordinate. This process can be completed in rooting formula. The experimental area can be extended if necessary. Since there are always two sides of the experiment area which are parallel to the axis, calculations of the extended area are same as $\triangle \mathrm{ABC}$ or $\triangle \mathrm{DBC}$.

Three-bonier measurement is:

$$
\begin{aligned}
& x_{0}=\frac{x_{O}+x_{P}+x_{Q}}{3} \\
& y_{0}=\frac{y_{O}+y_{P}+y_{Q}}{3}
\end{aligned}
$$

Where $\left(x_{0}, y_{0}\right)$ is coordinates of unknown point; $\left(x_{O}, y_{O}\right),\left(x_{P}, y_{P}\right)$ and $\left(x_{Q}, y_{Q}\right)$ are coordinates of the three points in Fig.1.

After obtaining the coordinates of unknown point, differential correction method will be used to amend coordinate values[11]. Differential correction method is:

$$
\begin{aligned}
& x_{M}=x_{0}+x_{e} \\
& y_{M}=y_{0}+y_{e}
\end{aligned}
$$

Where $\left(x_{e}, y_{e}\right)$ is differential correction factor, which is derived from experimental data of physical experimental platform.

A flowchart of isosceles right triangle layout location algorithm is shown in Fig.2.

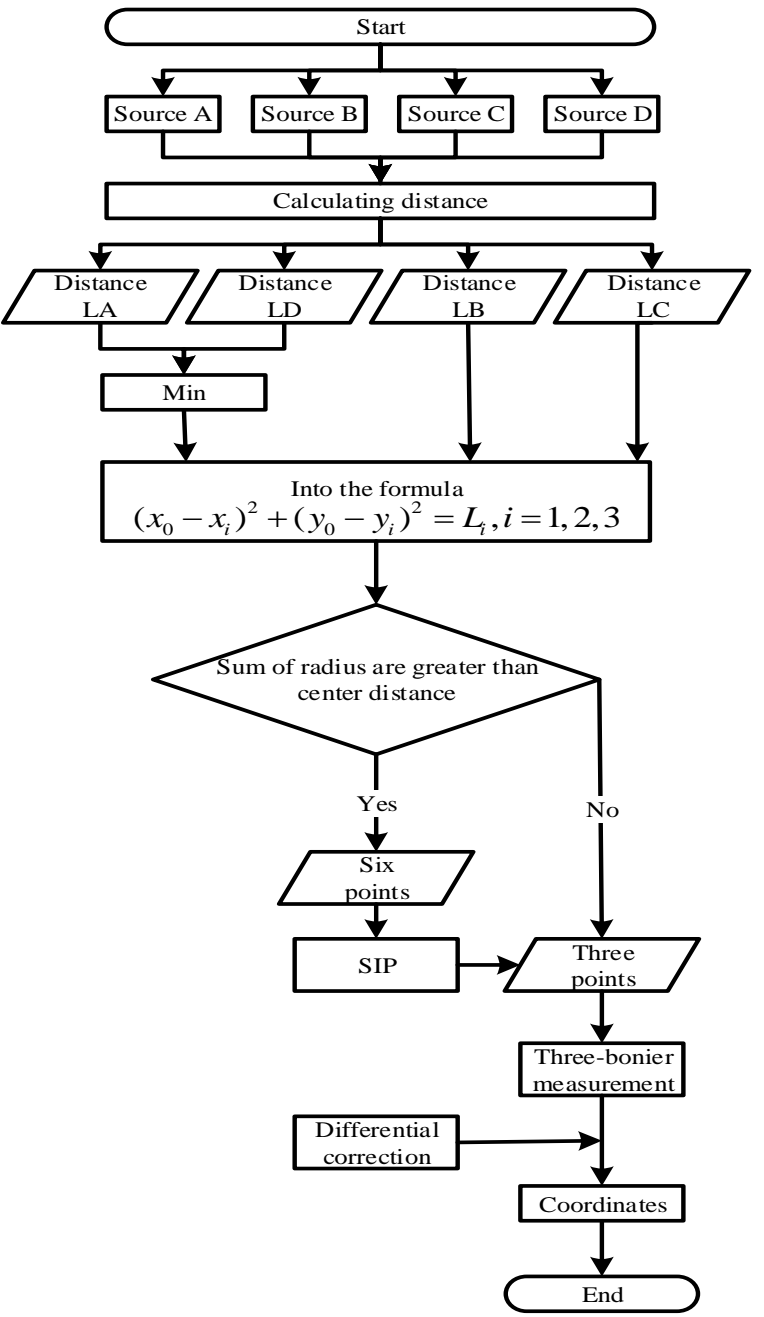

Figure 2. A flowchart of isosceles right triangle layout location algorithm. 


\section{B. Simulation Results}

\section{1) Model validation}

The given point's coordinates are $(2,2)$, the location results of the model are shown in Fig. 3 below:

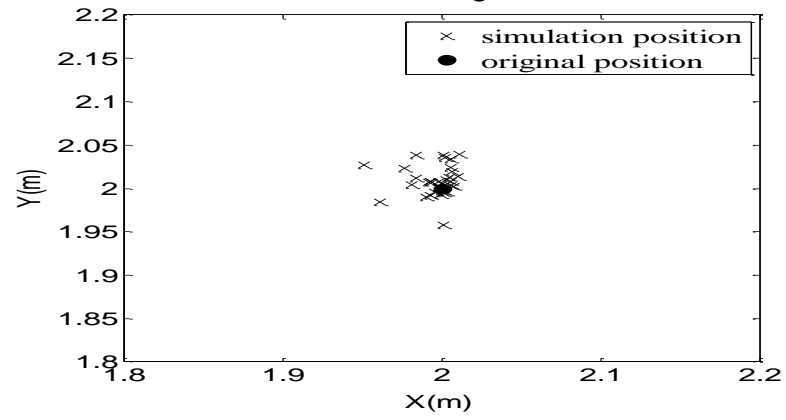

Figure 3. Repeatedly location results of one point with isosceles right triangle layout location algorithm.

As the Fig.3 shows, the results of location are $(2 \pm 0.02,2 \pm 0.02)$. The average error is $1 \%$. The worst error is $3 \%$. Experimental results show that the location model is acceptable.

2) Comparison of two kinds of layouts

The given point's coordinates are $(2,2)$. The given point is located 500 times with both layouts under the same experimental conditions, and then take the average as the final results. Results of isosceles triangle layout are $(2.01,2.02)$, and results of equilateral triangle layout are $(1.98,2.01)$. There is no obvious difference between location results of both layouts, so both of layouts will be verified on a hardware platform in the next section.

\section{APPLICATIONS BASED ON ARDUINO}

\section{A. Experiment Platform}

Arduino is a great and open-source electronic development platform[12]. Low-cost microprocessor (ATmega8 or ATmega128) makes it simple, cheap and approachable[14]. Therefore, Arduino is selected to build hardware platform.

In order to verify the proposed modified layout, a hardware platform is built. The hardware platform is composed of four routers and a receiver (including an Arduino UNO and a CC3000 WIFI Shield). Hardware schematic is shown in Fig.4.

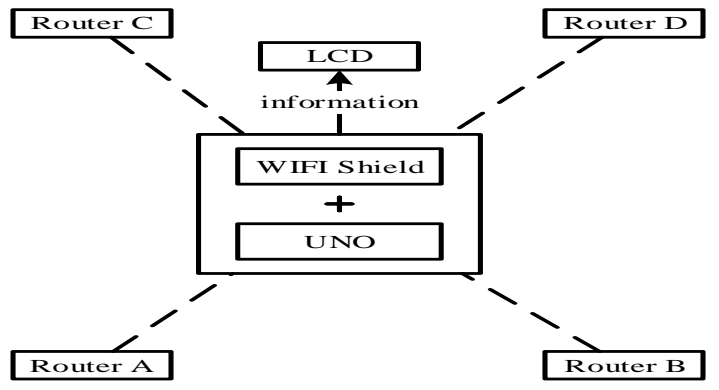

Figure 4. Hardware schematic

\section{B. Experiment}

The experimental area is shown in Fig.1. Four routers are placed at the four vertices of the square.

First, the relationship formula is obtained, which is shown in formula 4 in section 2 , between the distance and the RSSI value. In the experimental area, eight different directions and different distances around the router are selected as experimental points. The values at experimental points are collected and processed, and unknown parameters will be obtained. Then, location experiments will be implemented. Results of both layouts will be compared.

\section{Result Analysis}

According to the experimental data, the fitting results is shown in Fig.5:

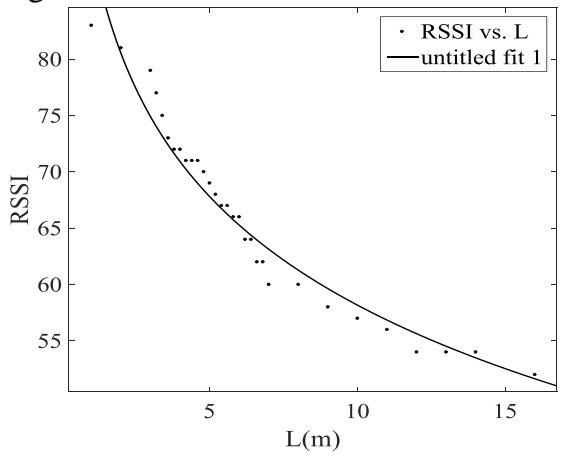

Figure 5. The fitting results of formula relationship of RSSI value and distance.

In the open experimental area, location experiments are carried out with both layouts.

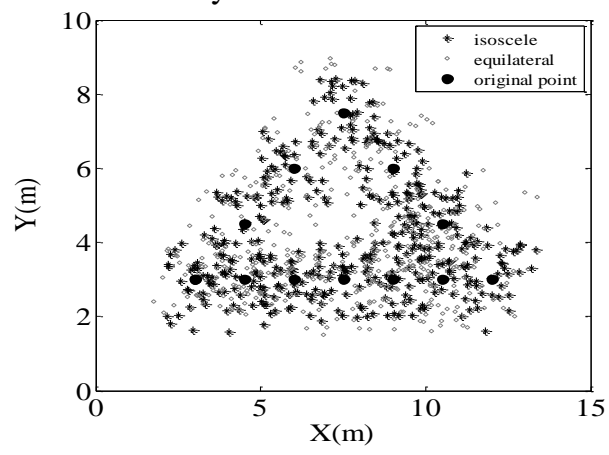

Figure 6. Comparative results of both layouts.

As shown in Fig.6, the black dots represent the experimental points' coordinates, which is the real position of the receiver. 'star' represent results of an isosceles triangle layout; diamond dots represent results of equilateral triangle layout. Each experimental point is respectively located 30 times with both layouts. Location error is distance between location coordinate and the black dots.

Results are as follow: location error of isosceles triangle layout is 1.28 meter; location error of equilateral triangle layout is 1.52 meter. Comparison of rapidity: 231 data are returned every $100 \mathrm{~s}$ in isosceles triangle layout; 216 data are returned every $100 \mathrm{~s}$ in equilateral triangle layout. 
Isosceles triangle layout is $0.3 \mathrm{~s}$ faster than equilateral triangle layout.

\section{SUMMARY}

In this paper, an improved isosceles triangle layout indoor location algorithm is proposed, which is more accurate and faster than equilateral triangle layout. Isosceles right triangle layout location algorithm is compact. In practical applications, it can increase the responding speed of hardware under certain conditions.

Faster location algorithm has a great significance for real-time location. Moreover, tracking and real-time navigation can be achieved with faster location algorithm.

\section{ACKNOWLEDGMENT}

This research was financially supported by the National Natural Science Foundation, project approval number is 61573035 .

\section{REFERENCES}

[1] Lanfang Xu: Three location model based on wireless LAN. Wu Han: Huazhong University of Science and Technology, 2008. (in Chinese)

[2] Yan Xu: Wireless sensor networks based on improved location algorithm of RSSI difference. Xiamen University, 2008, 47(3):361364. (in Chinese)

[3] Yan Zhou: Cyberspace location algorithm based on RSSI in wireless sensor. Journal on Communications, 2009, 30(6):76-79. (in Chinese)
[4] Zhenqiang Wang: The improved location algorithm based on RSSI difference. Communication Technology Daily, 2011, 44(7):78-80. (in Chinese)

[5] Xin Yao: UAV research to improve the targeting accuracy of the method. Ships in electrical engineering newspaper, 2011, 31(10):5861. (in Chinese)

[6] Xinyu Yang: An improved weighted centroid location algorithm. Journal of xi 'an jiaotong university, 2010, 44(8):2-4. (in Chinese)

[7] Eladio Martin. Precise Indoor Location Using Smart Phones Association for Computing Machinery, 2010, 10(1):25-29.

[8] Zhixian Liu: Indoor location algorithm based on RSSI. Guangxi Academy of Newspaper, 2015, 31(1):69-72. (in Chinese)

[9] Anindya S. Paul. WIFI Based Indoor Location and Tracking Using SIGMA-POINT Kalman Filtering Methods. USA: Oregon Health and Science University, 2006.

[10] J. Krumm and E. Horvitz, Locadio: Inferring motion and location from WIFI signal strengths, in Proceedings of International Conference on Mobile and Ubiquitious Systems: Networking and Services (MobiQuitous 04), 2004.

[11] Jin Xu: Algorithm of wireless location DGPS based on RSSI Instrument technique and sensor 2014, 5(1):76-79. (in Chinese)

[12] Hao Zheng: Design and implementation Bluetooth communication system based on Arduino/Android. Wu Han: Hubei University, 2012. (in Chinese)

[13] Tong Li: Design for Portable Visibility Meter based on Arduino. Nan Jing: Nanjing University of Science and Technology, 2013. (in Chinese)

[14] Nan Yang: Intelligent product prototype design and research based on Arduino. Wuxi: Jiang Nan university, 2014. (in Chinese) 